\title{
Numerical Analysis on Effective Mass and Traps Density Dependence of Electrical Characteristics of a-IGZO Thin-Film Transistors
}

\author{
Jihwan Park ${ }^{1}$, Do-Kyung Kim ${ }^{1}$, Jun-Ik Park ${ }^{1}$, In Man Kang ${ }^{1}$, Jaewon Jang ${ }^{1} \mathbb{D}$, Hyeok Kim ${ }^{2}$, \\ Philippe Lang ${ }^{3}$ and Jin-Hyuk Bae ${ }^{1, *(\mathbb{C})}$ \\ 1 School of Electronics Engineering, Kyungpook National University, Daegu 41566, Korea; \\ nrnr14@naver.com (J.P.); kdk7362@naver.com (D.-K.K.); rudiny7144@naver.com (J.-I.P.); \\ imkang@ee.knu.ac.kr (I.M.K.); j1jang@knu.ac.kr (J.J.) \\ 2 Department of Electrical and Computer Engineering, University of Seoul, Seoul 02504, Korea; \\ fomalhout@gmail.com \\ 3 ITODYS CNRS UMR 7086, Université Paris Diderot (Paris7), 15 rue Jean-Antoine de Baï, CEDEX 13, \\ 75205 Paris, France; lang@univ-paris-diderot.fr \\ * Correspondence: jhbae@ee.knu.ac.kr; Tel.: +82-53-950-7222
}

Received: 10 December 2019; Accepted: 6 January 2020; Published: 8 January 2020

\begin{abstract}
We have investigated the effect of electron effective mass $\left(m_{\mathrm{e}}{ }^{*}\right)$ and tail acceptor-like edge traps density $\left(N_{\mathrm{TA}}\right)$ on the electrical characteristics of amorphous-InGaZnO (a-IGZO) thin-film transistors (TFTs) through numerical simulation. To examine the credibility of our simulation, we found that by adjusting $m_{\mathrm{e}}{ }^{*}$ to 0.34 of the free electron mass $\left(\mathrm{m}_{\mathrm{o}}\right)$, we can preferentially derive the experimentally obtained electrical properties of conventional a-IGZO TFTs through our simulation. Our initial simulation considered the effect of $m_{\mathrm{e}}{ }^{*}$ on the electrical characteristics independent of $N_{\mathrm{TA}}$. We varied the $m_{\mathrm{e}}{ }^{*}$ value while not changing the other variables related to traps density not dependent on it. As $m_{\mathrm{e}}{ }^{*}$ was incremented to $0.44 \mathrm{~m}_{\mathrm{o}}$, the field-effect mobility $\left(\mu_{\mathrm{fe}}\right)$ and the on-state current $\left(I_{\text {on }}\right)$ decreased due to the higher sub-gap scattering based on electron capture behavior. However, the threshold voltage $\left(V_{\mathrm{th}}\right)$ was not significantly changed due to fixed effective acceptor-like traps $\left(N_{\mathrm{TA}}\right)$. In reality, since the magnitude of $N_{\mathrm{TA}}$ was affected by the magnitude of $m_{\mathrm{e}}{ }^{*}$, we controlled $m_{\mathrm{e}}{ }^{*}$ together with $N_{\mathrm{TA}}$ value as a secondary simulation. As the magnitude of both $m_{\mathrm{e}}{ }^{*}$ and $N_{\mathrm{TA}}$ increased, $\mu_{\mathrm{fe}}$ and Ion deceased showing the same phenomena as the first simulation. The magnitude of $V_{\text {th }}$ was higher when compared to the first simulation due to the lower conductivity in the channel. In this regard, our simulation methods showed that controlling $m_{\mathrm{e}}{ }^{*}$ and $N_{\mathrm{TA}}$ simultaneously would be expected to predict and optimize the electrical characteristics of a-IGZO TFTs more precisely.
\end{abstract}

Keywords: a-IGZO thin-film transistors; effective mass; numerical simulation; electrical characteristics; scattering

\section{Introduction}

Metal-oxide semiconductor-based thin-film transistors (TFTs) have been highlighted as the future technology for achieving organic light-emitting diode (OLED) and micro LED backplane applications for high-resolution, flexibility, low temperature processes, commercial display, and low power consumption [1,2]. In particular, amorphous indium-gallium-zinc-oxide (a-IGZO) has been identified as a promising candidate due to the high mobility, flexibility, low-temperature processing capability, uniformity, high on-state/off-states current ratio $\left(I_{\mathrm{on}} / I_{\mathrm{off}}\right)$, and easily controlled electrical characteristics by changing its chemical composition [3,4]. In order to take advantage of these properties, properties such as orbital superposition, oxygen vacancy states $\left(V_{\mathrm{o}}\right)$, peroxide states, and hydrogen 
complex had been demonstrated [5-8]. Among these, the effective mass $\left(m_{\mathrm{r}}^{*}\right)$ is important for the improvement of the hall mobility in the oxide semiconductor-based TFT [9-11]. However, it is difficult to understand the effect of $m_{\mathrm{r}}{ }^{*}$ on carrier transport because of the compensating potential energy caused due to internal force in the lattice [12]. Thus, it is essential for simulation studies to analyze and understand the effect of $m_{\mathrm{r}}$ * Simulation of a-IGZO TFTs is difficult when compared to c-Si-based metal oxide semiconductor field-effect transistors (MOSFETs) because of the need to consider many electrical parameters and chemical properties such as their amorphous phase, chemical properties, and ionic bonding properties [5-8]. Nevertheless, the simulation studies related to the electrical characteristics of a-IGZO TFTs have been studied in detail [13-15]. By applying these studies and theories, we could understand the effects of $m_{\mathrm{r}}{ }^{*}$ in a-IGZO TFTs. Therefore, it is necessary to study the change in the electrical characteristic due to the change in $m_{\mathrm{r}}{ }^{*}$. Thus, further simulation-based research was required to fully identify the mechanisms that control the electrical characteristics of a-IGZO TFTs.

In this work, we have investigated the electron effective mass $\left(m_{\mathrm{e}}^{*}\right)$ and tail acceptor-like edge traps density $\left(N_{\mathrm{TA}}\right)$ in the sub-gap density of states (DOS) using the technology computer-aided design (TCAD) simulations. At $m_{\mathrm{e}}{ }^{*}$ of 0.34 free electron mass $\left(\mathrm{m}_{\mathrm{o}}\right)$ [5], we can preferentially derive the very similar simulated results as the electrical characteristics of experimental a-IGZO TFTs [3]. After optimizing the parameters utilized to model results similar to the experiment, we controlled $m_{\mathrm{e}}{ }^{*}$ to analyze the effects of $m_{\mathrm{e}}{ }^{*}$ on the electrical characteristics of the a-IGZO TFT through energy band analysis. By changing only $m_{\mathrm{e}}$, the effect on various electrical characteristics such as field-effect mobility $\left(\mu_{\mathrm{fe}}\right)$, on-state current $\left(I_{\mathrm{on}}\right)$, and threshold voltage $\left(V_{\mathrm{th}}\right)$ changed, since the probability of electron capture also changed. Furthermore, in order to satisfy the boundary condition (BC), $N_{\mathrm{TA}}$ was varied according to $m_{\mathrm{e}}$. As a result, we obtained the electrical characteristics varied as a function $m_{\mathrm{e}}$ * and $N_{\mathrm{TA}}$ and analyzed the effective carrier mechanism related to acceptor-like traps. Therefore, it is expected that this work could be utilized to obtain the optimized electrical performance of a-IGZO TFTs for next-generation backplane applications.

\section{Simulation Methodology and Semiconductor Mechanisms}

The hall mobility property of oxide semiconductors can be explained by the orbital superposition $[5,10]$. These can vary depending on the oxide semiconductor material combination and chemical composition ratio [11]. In addition, it can be explained by varying the effective mass [12]. The effective mass is defined by quantum mechanics:

$$
\nabla^{2} \psi+\frac{2 \mathrm{~m}_{\mathrm{o}}}{\hbar}[P-V(\mathrm{r})]=0 .
$$

$P$ is the momentum energy, and $V(r)$ is the potential energy in the lattice. The potential energy is very difficult to calculate due to its relation to the internal force in the lattice. Thus, potential energy can be neglected for out simulation. We introduced a new mass concept $m_{\mathrm{r}}{ }^{*}$ instead of $\mathrm{m}_{\mathrm{o}}$ for energy preservation law, with the relationship of $\frac{1}{m_{\mathrm{r}}^{*}}=\frac{1}{m_{\mathrm{e}}^{*}}+\frac{1}{m_{\mathrm{h}}^{*}}$ :

$$
\nabla^{2} \psi+\frac{2 m_{\mathrm{r}}^{*} P}{\hbar}=0
$$

In the case of a-IGZO TFTs, we can approximate $\frac{1}{m_{\mathrm{r}}^{*}} \cong \frac{1}{m_{\mathrm{e}}^{*}}$ because of high values of hole effective mass $\left(m_{\mathrm{h}}{ }^{*}\right)$. Thus, $m_{\mathrm{e}}$ * is a very important factor to understand the electrical feature of a-IGZO TFTs. In order to fully understand the effect of $m_{\mathrm{e}}{ }^{*}$, we simulated the a-IGZO TFT by controlling $m_{\mathrm{e}}{ }^{*}$ and analyzed it using energy band analysis.

Figure 1a displays a schematic of the structure of a-IGZO-based bottom gate (BG)-top contact (TC) TFT. The gate (G), gate insulator (GI), active layer, and source (S) and drain (D) materials are n-polysilicon, $\mathrm{SiO}_{2}$, a-IGZO, and aluminum, respectively. The GI and a-IGZO active layer have a thickness of 100 and $20 \mathrm{~nm}$, respectively. The a-IGZO width (W) and length (L) are 180 and $30 \mu \mathrm{m}$. 
Figure $1 \mathrm{~b}$ schematically illustrates the sub-gap DOS distribution in relation to $m_{\mathrm{r}}{ }^{*}$ in a-IGZO TFTs. Both $N_{\mathrm{TA}}$ and the conduction-band DOS $\left(D_{\mathrm{C}}(E)\right)$, and tail donor-like edge traps density $\left(N_{\mathrm{TD}}\right)$ and valence-band DOS $\left(D_{\mathrm{V}}(E)\right)$ are theoretically continuous at boundary condition (BC) [16]. In addition, the Madelung potential could lead to a metastable condition. Therefore, $V_{\mathrm{O}}$ could form near the conduction band $\left(E_{C}\right)$ when the device is operated. Figure 1c presents a bonding model and the schematic of the energy band diagram in the a-IGZO TFT. When the gate voltage is applied, electrons are generated and operated to accumulation mode by $V_{\mathrm{O}}$ and oxygen interstitials $\left(I_{\mathrm{O}}{ }^{-2}\right)$ because of the Madelung potential and peroxide model [5,7].
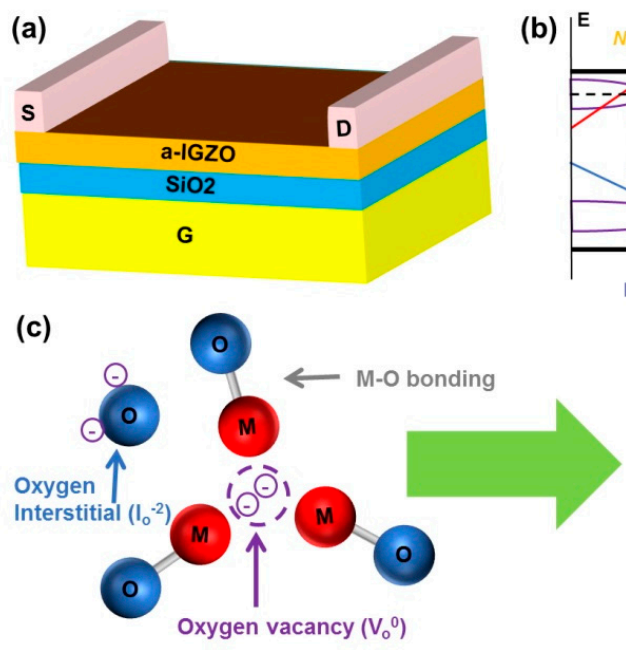

(b)

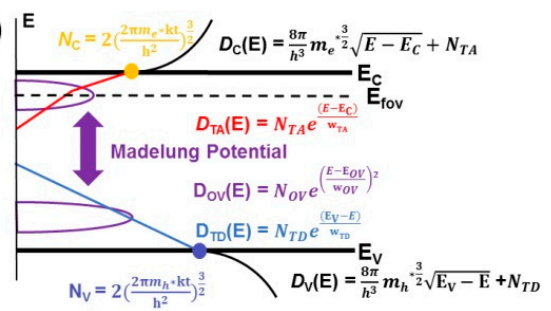

Figure 1. (a) schematic of the (amorphous-indium-gallium-zinc-oxide) a-IGZO (bottom gate-top contact) BG-TC structure. (b) Sub-gap (density of states) DOS distribution in the a-IGZO (thin-film transistor) TFT. (c) Bonding model and energy-band diagram for the a-IGZO TFT.

Figure 2a shows a cross-sectional view of the a-IGZO-based BC-TG TFT. We applied the interface traps model of Heiman $[17,18]$. Figure $2 b$ shows the sub-gap DOS distribution designed to match the RF-sputtered a-IGZO TFTs in terms of their electrical characteristics (RF sub-gap DOS) via sub-gap DOS engineering mechanism $[13,15]$.

(a)

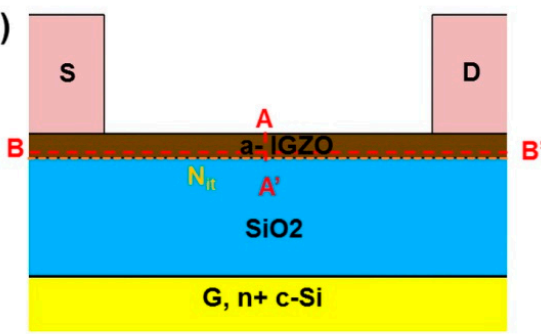

(c)

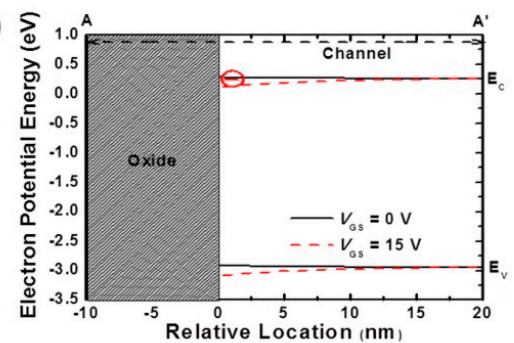

(b)

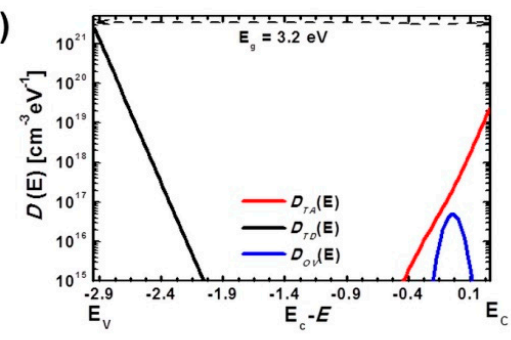

(d)

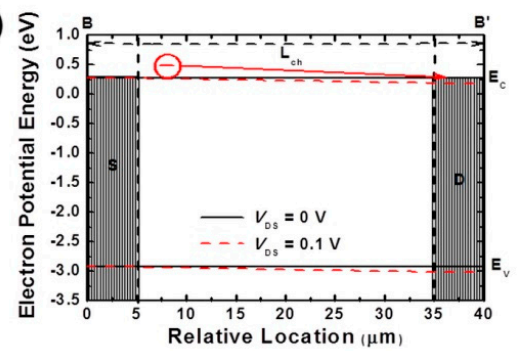

Figure 2. (a) cross-sectional view of a-IGZO TFT; (b) sub-gap DOS distribution for match (radio frequency) RF-sputtering a-IGZO BG-TC TFTs; (c) energy band diagram by cutline range of A to $\mathrm{A}^{\prime}$; (d) energy band diagram by cutline range of $B$ to $\mathrm{B}^{\prime}$. 
The total acceptor-like DOS $\left(D_{\mathrm{TA}}(E)\right)$, total donor-like DOS $\left(D_{\mathrm{TD}}(E)\right)$, and $V_{\mathrm{O}} \mathrm{DOS}\left(D_{\mathrm{OV}}(E)\right)$ have the properties of acceptor-like traps, donor-like traps, and $D_{\mathrm{OV}}(E)$, respectively. In practice, both $N_{\mathrm{TA}}$ and $N_{\mathrm{TD}}$ in the sub-gap DOS increase because of the presence of process stress and hydrogen defect states $[5,8]$. In this study, the RF sub-gap DOS design parameters were selected to match the RF-sputtered transfer characteristics of the a-IGZO TFTs as presented below in Table 1.

Table 1. Simulation parameters for the a-IGZO TFT.

\begin{tabular}{|c|c|c|c|}
\hline Parameter & Value & Unit & Description \\
\hline$E_{\mathrm{g}}$ & 3.2 & $\mathrm{eV}$ & Bandgap of a-IGZO \\
\hline$\chi_{\mathrm{s}}$ & 4.16 & $\mathrm{eV}$ & Affinity of a-IGZO \\
\hline$\varepsilon_{\mathrm{Ox}}$ & 3.9 & $\varepsilon_{0}$ & $\mathrm{SiO}_{2}$ dielectric constant \\
\hline$N_{\mathrm{OV}}$ & $5 \times 10^{16}$ & $\mathrm{~cm}^{-3} \mathrm{eV}^{-1}$ & Gauss Donor-like edge traps density of $D_{\mathrm{OV}}(E)$ \\
\hline$W_{\mathrm{OV}}$ & 0.08 & $\mathrm{eV}$ & Characteristic decay energy of $D_{\mathrm{OV}}(E)$ \\
\hline$E_{\mathrm{OV}}$ & 3.0 & $\mathrm{eV}$ & Energy peak of $D_{\mathrm{OV}}(E)$ \\
\hline$N_{\mathrm{TA}}$ & $2.4 \times 10^{19}$ & $\mathrm{~cm}^{-3} \mathrm{eV}^{-1}$ & Tail acceptor-like edge traps density \\
\hline$W_{\mathrm{TA}}$ & 0.06 & $\mathrm{eV}$ & Characteristic decay energy of $D_{\mathrm{TA}}(E)$ \\
\hline$W_{\mathrm{GA}}$ & 0.28 & $\mathrm{eV}$ & Characteristic decay energy of $D_{\mathrm{GA}}(E)$ \\
\hline$W_{\mathrm{TD}}$ & 0.08 & $\mathrm{eV}$ & Characteristic decay energy of $D_{\mathrm{TD}}(E)$ \\
\hline$N_{\text {it }}$ & $1.1 \times 10^{11}$ & $\mathrm{~cm}^{-3} \mathrm{eV}^{-1}$ & Effective interface traps density \\
\hline$d$ & 2 & $\mathrm{~nm}$ & Interface trap depth between semiconductor and gate insulator \\
\hline$c_{D n}$ & $1 \times 10^{-16}$ & $\mathrm{~cm}^{2}$ & Electron capture cross-section of the donor-like trap \\
\hline$c_{D p}$ & $1 \times 10^{-14}$ & $\mathrm{~cm}^{2}$ & Hole capture cross-section of the donor-like trap \\
\hline$c_{\text {An }}$ & $1 \times 10^{-14}$ & $\mathrm{~cm}^{2}$ & Electron capture cross-section of the acceptor-like trap \\
\hline$c_{\text {Ap }}$ & $1 \times 10^{-16}$ & $\mathrm{~cm}^{2}$ & Hole capture cross-section of the acceptor-like trap \\
\hline
\end{tabular}

Figure 2c,d present an energy band diagram of the a-IGZO TFT with an A to $\mathrm{A}^{\prime}$ vertical cutline and a B to $B^{\prime}$ lateral cutline. This illustration can not only explain the mechanism of field-effect TFTs, but also can be used to predict the electron distribution when the device operates according to Poisson's Equation from Labels (3) to (6):

$$
\begin{gathered}
\frac{d F}{d x}=\frac{q\left(D_{\mathrm{OV}}^{+2}(E)-2 e-D_{\mathrm{TA}}(E)\right)}{\epsilon_{s} \epsilon_{o}}, \\
\frac{d F}{d x}=\frac{q\left(2 N_{\mathrm{OV}}^{+2}-N_{\mathrm{TA}}^{-}\right)}{\epsilon_{s} \epsilon_{o}}, \\
F(x)=-\frac{q\left(2 N_{\mathrm{OV}}^{+2}-N_{\mathrm{TA}}^{-}\right)}{\epsilon_{s} \epsilon_{o}}\left(W_{\mathrm{D}}-x\right), \\
V(x)=-\frac{q\left(2 N_{\mathrm{OV}}^{+2}-N_{\mathrm{TA}}^{-}\right)}{2 \epsilon_{s} \epsilon_{o}}\left(W_{\mathrm{D}}-x\right)^{2},
\end{gathered}
$$

where $\epsilon_{s}$ is the a-IGZO dielectric constant set at 10 in this study, $\epsilon_{o}$ is the vacuum permittivity, $F$ is the electric field, $q$ is the elementary charge, $W_{\mathrm{D}}$ is the depletion width, and $x$ is the relative location. The energy band analysis could observe the effect of traps through the variation of energy when an electron is working. Figure 3 shows the relationship of $m_{\mathrm{r}}{ }^{*}$ to the design parameters: $N_{\mathrm{TA}}, N_{\mathrm{TD}}$, thermal velocity $\left(v_{\mathrm{t}}\right)$, the lifetime related to trap site $\left(\tau_{\mathrm{t}}\right)$ at the $N_{\mathrm{OV}}$ (donor-like edge traps density of $\left.D_{\mathrm{OV}}(E)\right)$, and the hall mobility $\left(\mu_{0}\right)$. These variables were calculated using formulas from Labels $(7)$ to (10), respectively [18,19]. At an $m_{\mathrm{e}}{ }^{*}$ of 0.34 and an $m_{\mathrm{h}}{ }^{*}$ of $21 \mathrm{~m}_{\mathrm{o}}[5,13]$, the following design parameters were obtained: a $N_{\mathrm{TA}}$ of $4.97 \times 10^{18} \mathrm{~cm}^{-3} \mathrm{eV}^{-1}$, a $N_{\mathrm{TD}}$ of $2.41 \times 10^{21} \mathrm{~cm}^{-3} \mathrm{eV}^{-1}$, a $v_{\mathrm{t}}$ of $2 \times 10^{7} \mathrm{cms}^{-1}$, a $\tau_{\mathrm{n}}$ of $10 \mathrm{~ns}$, a $\tau_{\mathrm{p}}$ of $2 \mathrm{~ns}$, and a $\mu_{0}$ of $22 \mathrm{~cm}^{2} \mathrm{~V}^{-1} \mathrm{~s}^{-1}$. As a result, we can completely obtain the transfer characteristics of the RF-sputtered a-IGZO BG-TC TFTs (Table 2). 

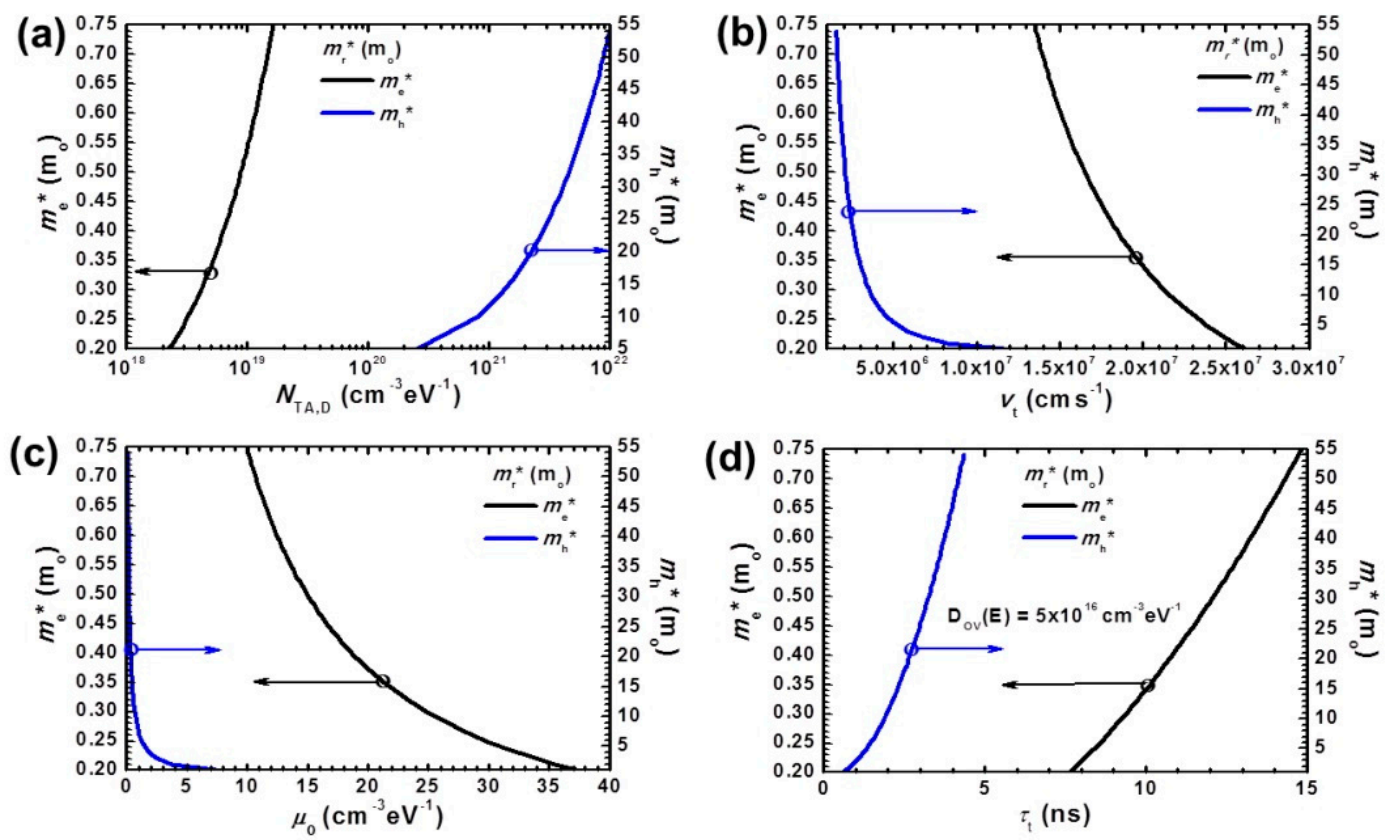

Figure 3. Distributions of (a) $N_{\mathrm{TA}}$ and $N_{\mathrm{TD}}$ (b) $v_{\mathrm{t}}$, and (c) $\mu_{0}$ according to $m_{\mathrm{r}}{ }^{*}$, (d) distributions of $\tau_{\mathrm{t}}$ according to $m_{\mathrm{r}}^{*}$ at the $D_{\mathrm{OV}}(E)=5 \times 16 \mathrm{~cm}^{-3} \mathrm{eV}^{-1}$.

Table 2. Comparison with good transfer characteristics of a-IGZO TFTs derived from previous experimental-based work via RF-sputtering [3] and those derived numerically form this work.

\begin{tabular}{ccccc}
\hline Comparison & $\boldsymbol{M}_{\mathbf{e}}\left(\mathbf{m}_{\mathbf{o}}\right)$ & $\begin{array}{c}\mu_{\mathbf{f e}} \\
\left(\mathbf{c m}^{\mathbf{2}} \mathbf{V}^{-\mathbf{1}} \mathbf{s}^{-\mathbf{1}}\right)\end{array}$ & $\boldsymbol{V}_{\mathbf{t h}} \mathbf{( V )}$ & $\boldsymbol{I}_{\mathbf{o n}} \mathbf{( A )}$ \\
\hline a-IGZO TFTs [3] & 0.34 & 12.9 & 3.1 & $\sim 10^{-6}$ \\
This work & 0.34 & 12.9 & 3.1 & $3 \times 10^{-6}$ \\
\hline
\end{tabular}

$$
\begin{gathered}
N_{\mathrm{C}, \mathrm{V}}=2\left(\frac{2 \pi m_{\mathrm{r}}^{*} k t}{h^{2}}\right)^{\frac{3}{2}}, \\
v_{\mathrm{t}}=\sqrt{\frac{3 k t}{m_{\mathrm{r}}^{*}}} \\
\tau_{\mathrm{t}}=\frac{1}{v_{\mathrm{t}} c_{\mathrm{n}, \mathrm{h}} N_{\mathrm{T}}(E)}, \\
\mu_{0}=\frac{q h}{12 \pi m_{\mathrm{r}}^{*} k t^{\prime}},
\end{gathered}
$$

in the above Equations, $k$ is the Boltzmann constant, and $t$ is room temperature of $300 \mathrm{~K} . c_{\mathrm{n}, \mathrm{p}}$ is the capture cross-section of electron and hole, $N_{\mathrm{T}}(E)$ is the trap-DOS in the active layer, and $h$ is the Planck constant $(2 \pi \hbar)$.

We assumed that $C_{\mathrm{An}}, C_{\mathrm{Ah}}, C_{\mathrm{Dh}}$, and $C_{\mathrm{Dn}}$ were $1 \times 10^{-14}, 1 \times 10^{-16}, 1 \times 10^{-16}$, and $1 \times 10^{-14} \mathrm{~cm}^{2}$, respectively, since the acceptor-like traps are negatively charged when occupied by an electron, and the donor-like traps are positively charged when emptied. We also assume a fully ionized $D_{\mathrm{OV}}(E)$ at the metastable condition. Thus, the electron concentration was calculated to be $1 \times 10^{17} \mathrm{~cm}^{-3}$ and $\left(E_{\mathrm{C}}\right.$ $-E_{\mathrm{f}}$ ) is calculated to be $0.1 \mathrm{eV}$ using Equation (9):

$$
\left(E_{\mathrm{C}}-E_{\mathrm{f}}\right)=k t \ln \left(\frac{N_{\mathrm{C}}}{2 N_{\mathrm{OV}}}\right) .
$$


The interface traps were modeled with the Heiman model because the electrical mechanisms between the GI and semiconductor layer are better expressed when the transient time is taken into consideration during device operation. The Heiman interface mechanism could be obtained from the following equations:

$$
\begin{gathered}
\frac{d N_{\mathrm{it}}^{-}}{d t}=N_{\mathrm{it}}\left[v_{\mathrm{tn}} c_{\mathrm{n}}(d)\left\{\mathrm{n}\left(1-f_{\mathrm{it}}\right)-f_{\mathrm{it}} n_{\mathrm{i}} e^{\frac{E_{\mathrm{t}}-E_{\mathrm{i}}}{k t}}\right\}-v_{\mathrm{th}} c_{\mathrm{h}}(\mathrm{d})\left\{\mathrm{h} f_{\mathrm{it}}-\left(1-f_{\mathrm{it}}\right) n_{\mathrm{i}} e^{\frac{E_{\mathrm{i}}-E_{\mathrm{t}}}{k t}}\right\}\right], \\
c_{\mathrm{n}}(d)=c_{\mathrm{n}} e^{-\left\{\frac{2 m_{\mathrm{e}}^{*}\left(E_{\mathrm{C}}-E_{\mathrm{it}}\right)}{\hbar^{2}}\right\} d,} \\
c_{\mathrm{h}}(d)=c_{\mathrm{h}} e^{-\left\{\frac{2 m_{\mathrm{h}}^{*}\left(E_{\mathrm{it}}-E_{\mathrm{V}}\right)}{\hbar^{2}}\right\} d,}
\end{gathered}
$$

where $N_{\mathrm{it}}$ is the effective interface traps density and $d$ is the interface traps density depth between a-IGZO and GI. For a more accurate simulation, we used Shockley-Read-Hall (SRH) recombination with trap-assisted tunneling [18,20]:

$$
R_{\mathrm{TAT}}=\frac{n_{\mathrm{i}}^{2}-n h}{\frac{\tau_{\mathrm{h}}}{1+E_{\mathrm{rh}}^{2}\left(\frac{1}{k t}-\frac{10}{3} E_{\mathrm{rh}}\left(\frac{2 m_{\mathrm{h}}^{*}}{q h \mathrm{~h} \mid}\right)\right.}\left(n+n_{\mathrm{i}} e^{\left(\frac{E_{\mathrm{T}}-E_{\mathrm{i}}}{k t}\right)}\right)+\frac{\tau_{\mathrm{n}}^{\frac{1}{2}}}{1+E_{\mathrm{rn}}^{2}\left(\frac{1}{k t}-\frac{10}{3} E_{\mathrm{rn}}\left(\frac{2 m_{\mathrm{e}}^{*}}{q h \mid \mathrm{H}}\right)^{\frac{1}{2}}\right.}\left(h+h_{\mathrm{i}} e^{\left(\frac{E_{\mathrm{i}}-E_{\mathrm{T}}}{k t}\right)}\right)},
$$

where $E_{\mathrm{rh}}$ is the hole tunneling range, $E_{\mathrm{rn}}$ is the electron tunneling range, and $E_{\mathrm{T}}$ is the trap energy level.

\section{Results and Discussion}

Figure 4a shows the drain current-gate voltage ( $\left.I_{\mathrm{DS}}-V_{\mathrm{GS}}\right)$ transfer characteristics at the RF sub-gap DOS with the variation of $m_{\mathrm{e}}{ }^{*}$ in the linear and saturation regions. As $m_{\mathrm{e}}$ increased up to $0.44 \mathrm{~m}_{\mathrm{o}}, \mu_{\mathrm{fe}}$ and $I_{\mathrm{on}}$ in the linear region fall to 10.6 and $2.7 \mu \mathrm{A}$, respectively. In order to analyze the effect of only changing $m_{\mathrm{e}}{ }^{*}$, we extracted a vertical energy band diagram at $V_{\mathrm{GS}}=15$ and $V_{\mathrm{DS}}=0.1 \mathrm{~V}$ using the simulation tools as shown in Figure $4 \mathrm{~b}$. As $m_{\mathrm{e}}{ }^{*}$ changed from 0.24 to $0.44 \mathrm{~m}_{\mathrm{o}}$, the electron potential energy also increases from -0.067 to $-0.049 \mathrm{eV}$. This meant that high $m_{\mathrm{e}}{ }^{*}$ electrons were more easily captured and hardly emitted when compared to low $m_{\mathrm{e}}{ }^{*}$ electrons. This was due to the negative charge of acceptor-like traps when holding the electrons, which could be explained using the Poisson's Equation. Therefore, the relationship between $m_{\mathrm{e}}{ }^{*}$ and $N_{\mathrm{TA}}$ is expressed as:

$$
m_{\mathrm{e}}^{*} \propto e_{\mathrm{t}} \propto \frac{1}{e_{\mathrm{e}}} .
$$

The $e_{\mathrm{t}}$ defines the probability that the electrons are trapped by $N_{\mathrm{TA}}$, and $e_{\mathrm{e}}$ defines the probability that the electrons escape $N_{\mathrm{TA}}$. It was utilized to study the effect of $m_{\mathrm{e}}{ }^{*}$ through energy band analysis.

However, it is necessary for $\mathrm{BC}$ to consider the variation of $N_{\mathrm{TA}}$ by $m_{\mathrm{e}}{ }^{*}$. as the $N_{\mathrm{TA}}$ would cause sub-gap scattering. The sub-gap scattering could be reinterpreted by the following Equations $[13,18]$ :

$$
\begin{gathered}
\frac{1}{\mu}=\frac{1}{\mu_{\mathrm{pn}}}+\frac{1}{\mu_{\mathrm{sg}}}+\frac{1}{\mu_{\mathrm{sf}}} \\
\mu_{\mathrm{sg}}=\frac{\mu_{0}}{1+\left[\left(D_{\mathrm{TA}}(E)+D_{\mathrm{OV}}(E)\right) /(\text { cubic })\right]^{a}}+\mathrm{b} .
\end{gathered}
$$



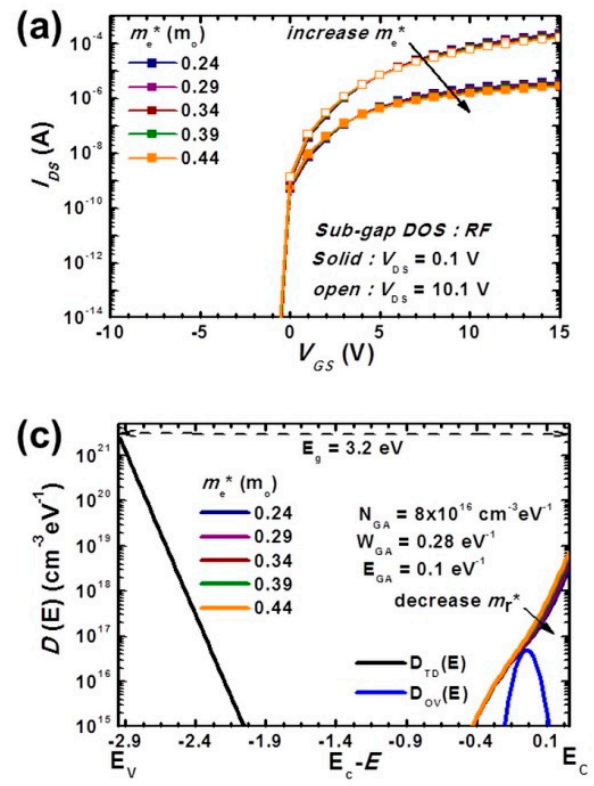

(b)

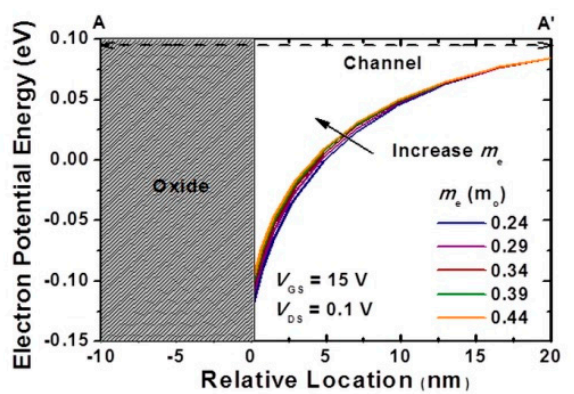

(d)

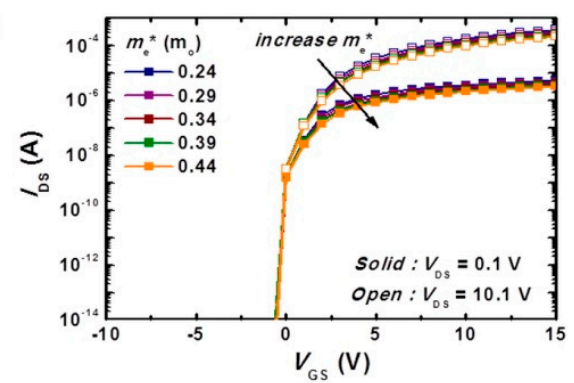

Figure 4. (a) transfer curve for an $m_{\mathrm{e}}{ }^{*}$ range of 0.24 to $0.44 \mathrm{~m}_{\mathrm{o}}$ at the RF sub-gap DOS, (b) vertical energy band diagram for a cutline range of $\mathrm{A}$ to $\mathrm{A}^{\prime}$ at $V_{\mathrm{GS}}=15 \mathrm{~V}$ and $V_{\mathrm{DS}}=0.1 \mathrm{~V}$, (c) Sub-gap DOS distribution theoretically satisfying the (boundary condition) $\mathrm{BC}$, (d) transfer curve for an $m_{\mathrm{e}}{ }^{*}$ range of 0.24 to $0.44 \mathrm{~m}_{\mathrm{o}}$ at the BC sub-gap DOS.

In the above equations, $\mu_{\mathrm{pn}}$ denotes phonon scattering due to lattice vibrations, $\mu_{s f}$ represents surface scattering due to interface traps, $\mu_{\mathrm{sg}}$ shows sub-gap scattering, the cubic is $D_{\mathrm{OV}}(E)$ and $D_{\mathrm{TA}}(E)$ per cubic centimeter, and $a$ and $b$ are experimental coefficients. Therefore, a-IGZO TFT could reinterpret the sub-gap scattering mechanism. Figure $4 \mathrm{c}$ shows that, by changing $m_{\mathrm{e}}{ }^{*}, N_{\mathrm{TA}}$ is theoretically is affected. The $N_{\mathrm{TA}}$ was increased from $2.95 \times 10^{18}$ to $7.32 \times 10^{18} \mathrm{~cm}^{-3} \mathrm{eV}^{-1}$ when the $m_{\mathrm{e}}{ }^{*}$ changed from 0.24 to $0.44 \mathrm{~m}_{\mathrm{o}}$. $W_{\mathrm{TA}}$ is not related to $m_{\mathrm{r}}{ }^{*}$ but rather related to amorphous randomness $[9,11]$, and $\mathrm{N}_{\mathrm{GA}}$ $(E)$ is not related to $m_{\mathrm{r}}{ }^{*}$ but rather deep traps density $[4,8]$. Thus, these values were maintained. Therefore, in order to accurately interpret the effect of electrical characteristics on $m_{\mathrm{r}}{ }^{*}, \mathrm{~W}_{\mathrm{TA}}$ and $\mathrm{N}_{\mathrm{GA}}$ (E) should maintain their values in this paper. As a result, we observed a high variation of transfer curve under the $V_{\text {th }}$ region at the BC sub-gap DOS as shown in Figure $4 \mathrm{~d}$. In order to obtain more detailed electrical performance trends, we used the $g_{\mathrm{m}}$ extraction method as below Equation $[3,5]$ :

$$
\begin{gathered}
I_{\mathrm{DS}}=\mu_{\mathrm{fe}, \text { lin }} \cdot C_{\mathrm{ox}}\left(\frac{W}{L}\right)\left(V_{\mathrm{GS}}-V_{\mathrm{th}}\right) V_{\mathrm{DS}}-\left(\frac{V_{\mathrm{DS}}^{2}}{2}\right)\left(\text { at } V_{\mathrm{GS}}-V_{\mathrm{th}}>V_{\mathrm{DS}}\right), \\
I_{\mathrm{DS}}=\mu_{\mathrm{fe}, \mathrm{sat}} \cdot C_{\mathrm{ox}}\left(\frac{W}{2 L}\right)\left(V_{\mathrm{GS}}-V_{\mathrm{th}}\right)^{2}\left(\text { at } V_{\mathrm{DS}} \geq V_{\mathrm{GS}}-V_{\mathrm{th}}\right) .
\end{gathered}
$$

Thus, $\mu_{\mathrm{fe}}$ and $V_{\text {th }}$ can be obtained using the following $g_{\mathrm{m}}$ extraction equation:

$$
\begin{gathered}
g_{\mathrm{m}}=\frac{\partial I_{\mathrm{DS}}}{\partial V_{\mathrm{GS}}}=\mu_{\mathrm{fe}, \text { lin }} \cdot C_{\mathrm{ox}}\left(\frac{W}{L}\right) V_{\mathrm{DS},} \\
\mu_{\mathrm{fe}, \mathrm{lin}}\left(V_{\mathrm{GS}}\right)=\frac{g_{\mathrm{m}}\left(V_{\mathrm{GS}}\right)}{C_{\mathrm{ox}} \cdot V_{\mathrm{DS}}}\left(\frac{L}{W}\right), \\
g_{\mathrm{m}}=\frac{\partial I_{\mathrm{DS}}^{\frac{1}{2}}}{\partial V_{\mathrm{GS}}}=\left(\mu_{\mathrm{fe}, \mathrm{sat}} \cdot C_{\mathrm{ox}}\left(\frac{W}{2 L}\right)\right)^{\frac{1}{2}}, \\
\mu_{\mathrm{fe}, \mathrm{sat}}=\frac{\left(g_{\mathrm{m}}\right)^{2}}{C_{\mathrm{ox}}}\left(\frac{2 L}{W}\right) .
\end{gathered}
$$


The $C_{\text {ox }}$ denotes the $\mathrm{SiO}_{2}$ capacitance.

Figure $5 \mathrm{a}$ shows $\mu_{\mathrm{fe}}$ by changing $m_{\mathrm{e}}{ }^{*}$ at the RF and BC sub-gap DOS distributions in the linear and saturation regions. As the $m_{\mathrm{e}}{ }^{*}$ increased from 0.24 to $0.44 \mathrm{~m}_{\mathrm{o}}, \mu_{\mathrm{fe}}$ decreased from 16.4 to $10.6 \mathrm{~cm}^{2} \mathrm{~V}^{-1} \mathrm{~s}^{-1}$ in the linear region, and from 17.1 to $10.8 \mathrm{~cm}^{2} \mathrm{~V}^{-1} \mathrm{~s}^{-1}$ in the saturation region at the RF sub-gap DOS distribution. At the BC sub-gap DOS distribution, as the $m_{\mathrm{e}}{ }^{*}$ increased from 0.24 to $0.44 \mathrm{~m}_{\mathrm{o}}, \mu_{\mathrm{fe}}$ also decreased from 18.5 to $11.7 \mathrm{~cm}^{2} \mathrm{~V}^{-1} \mathrm{~s}^{-1}$ in the linear region, and from 19.7 to $12.1 \mathrm{~cm}^{2} \mathrm{~V}^{-1} \mathrm{~s}^{-1}$ in the saturation region. It was found that the $\mathrm{BC}$ sub-gap DOS distribution exhibited higher $\mu_{\mathrm{fe}}$ than the RF sub-gap DOS distribution because of relatively high $N_{\mathrm{TA}}$. Figure $5 \mathrm{~b}$ shows the variation of $I_{\mathrm{on}}$ with $m_{\mathrm{e}}{ }^{*}$ at the RF and BC sub-gap DOS distributions in the linear and saturation regions. As $m_{\mathrm{e}}{ }^{*}$ increased from 0.24 to $0.44 \mathrm{~m}_{\mathrm{o}}, I_{\mathrm{on}}$ decreased from 4.0 to $2.7 \mu \mathrm{A}$ in the linear region and from 0.23 to $0.16 \mathrm{~mA}$ in the saturation region at the RF sub-gap DOS distribution. At the BC sub-gap DOS distribution, as $m_{\mathrm{e}}{ }^{*}$ increased, $I_{\mathrm{on}}$ also decreased from 5.3 to $3.2 \mu \mathrm{A}$ in the linear region, and from 0.36 to $0.21 \mathrm{~mA}$ in the saturation region. It was found that the BC sub-gap DOS distribution exhibited higher $I_{\mathrm{on}}$ than RF sub-gap DOS distribution also because of the relatively high $N_{\mathrm{TA}}$. The high $N_{\mathrm{TA}}$ distribution in the a-IGZO TFTs was caused by the increasing probability of capture electron and sub-gap scattering. Figure $5 \mathrm{c}$ shows the change in $V_{\text {th }}$ with the variation of $m_{\mathrm{e}}{ }^{*}$ at the RF and BC sub-gap DOS distributions in the linear and saturation regions. As $m_{\mathrm{e}}{ }^{*}$ increased from 0.24 to $0.44 \mathrm{~m}_{\mathrm{O}}$, $V_{\text {th }}$ decreased from 3.4 to $2.9 \mathrm{~V}$ in the linear region, and from 3.3 to $2.6 \mathrm{~V}$ in the saturation region at the RF sub-gap DOS distribution. Unexpectedly, the $V_{\text {th }}$ decreased in the transfer curve at the RF sub-gap DOS distribution, because the $x$-axis intersection had negative shifted with decreasing slope, as evident from the $g_{\mathrm{m}}$ extraction equation. However, intrinsic $V_{\text {th }}$ would have a slight increase as noticed through energy band analysis. At the BC sub-gap DOS distribution, as $N_{\mathrm{TA}}$ increases from 2.95 $\times 10^{18}$ to $7.32 \times 10^{18} \mathrm{~cm}^{-3} \mathrm{eV}^{-1}$ by varying $m_{\mathrm{e}}{ }^{*}$ from 0.24 to $0.44 \mathrm{~m}_{\mathrm{o}}, V_{\text {th }}$ increased from 0.7 to $1.5 \mathrm{~V}$ in the linear region, and from 0.7 to $1.3 \mathrm{~V}$ in the saturation region. It was found that $N_{\mathrm{TA}}$ affected $V_{\text {th }}$ more than $m_{\mathrm{e}}{ }^{*}$. This could be utilized to predict the practical tendency of $V_{\mathrm{th}}$ through optimizing $m_{\mathrm{e}}{ }^{*}$.

(a)

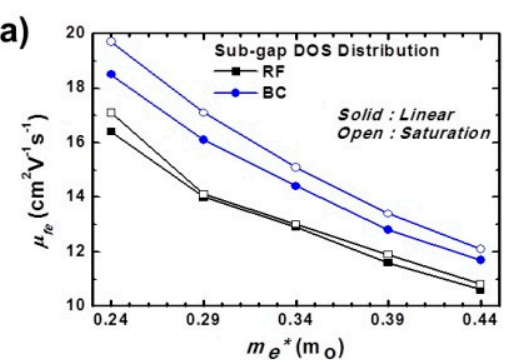

(c)

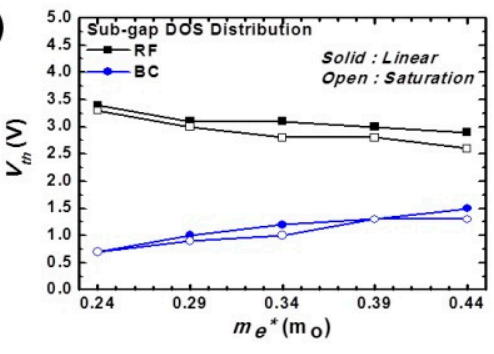

(b)

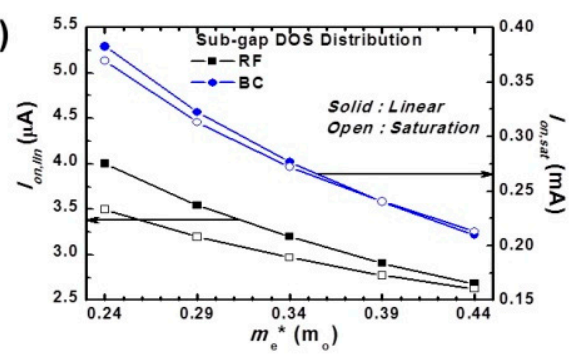

(d)

Applied Voltage Bias

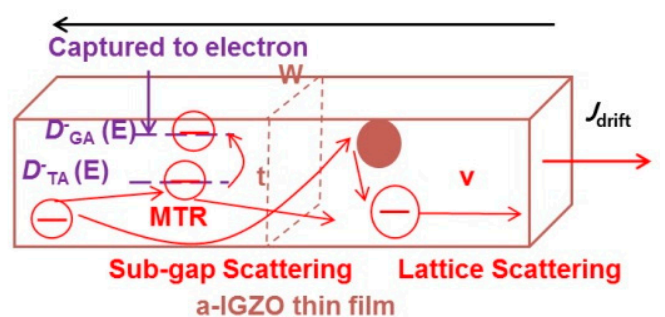

Figure 5. (a) $\mu_{\mathrm{fe}}$ versus $m_{\mathrm{e}}{ }^{*}$, (b) $I_{\text {on }}$ versus $m_{\mathrm{e}}{ }^{*}$, and (c) $V_{\text {th }}$ versus $m_{\mathrm{e}^{*}}$ at the RF and BC sub-gap DOS distribution in the linear and saturation region, (d) schematic of the (multiple trapping and release) MTR, sub-gap scattering, lattice scattering, and trapping electron mechanisms in the a-IGZO TFT.

To illustrate this trend, we reinterpreted the conductivity mechanism [21]. It can be expressed to drift drain current density $\left(J_{n, \text { drift }}\right)$ as

$$
J_{\mathrm{n}, \mathrm{drift}}=q\left(n-n_{\mathrm{t}}\right) u_{\mathrm{fe}} F
$$


Thus, the conductivity is

$$
\sigma=q\left(n-n_{\mathrm{t}}\right) u_{\mathrm{fe}}
$$

It is hypothesized that the observed positive shift in $V_{\text {th }}$ at the BC sub-gap DOS was because of the low conductivity, which caused difficulty in channel formation through the electron capture by $N_{\text {TA }}$. To understand it in more detail, Figure $5 \mathrm{~d}$ shows the mechanism of multiple trapping and release (MTR) [22], sub-gap scattering, and low conductivity effect of $m_{\mathrm{e}}{ }^{*}$. Therefore, $m_{\mathrm{e}}{ }^{*}$ is a very important parameter because it affects $N_{\mathrm{TA}}$, and has a major influence on the electrical performances in a-IGZO TFT. In addition, we would expect greater improvements in device performance to arise from appropriate $m_{\mathrm{e}}{ }^{*}$ design and sub-gap engineering compared with conventional a-IGZO TFTs.

\section{Conclusions}

In this work, the a-IGZO-based BG-TC TFT was designed through experimental-based a-IGZO TFTs using TCAD simulations. Based on the parameters optimized to mimic experimental results, we only changed $m_{\mathrm{e}}{ }^{*}$ from 0.24 to $0.44 \mathrm{~m}_{\mathrm{o}}$ to study its effects. For low $m_{\mathrm{e}}{ }^{*}, \mu_{\mathrm{fe}}$, and $I_{\text {on }}$ were higher because of a lower probability of electron capture by $N_{\mathrm{TA}}$ through energy band analysis. Furthermore, we have investigated the influence of $m_{\mathrm{e}}{ }^{*}$ and $N_{\mathrm{TA}}$ on the BC sub-gap DOS. As a result, we had reinterpreted the effects of sub-gap scattering, low conductivity, and the relationship between $m_{\mathrm{e}}{ }^{*}$ and $N_{\mathrm{TA}}$ through energy band analysis and semiconductor mechanism. Thus, we were able to obtain the tendencies of $\mu_{\mathrm{fe}}, I_{\mathrm{on}}$, and $V_{\text {th }}$ electrical characteristics of a-IGZO TFT. In addition, our model could be utilized to predict the limit of theoretical electrical characteristics according to $m_{\mathrm{e}}{ }^{*}$ of a-IGZO TFT. Therefore, this study would be able to provide valuable information for the design of backplane unit devices for high-resolution applications.

Author Contributions: The manuscript was written and the software validated by J.P. Formal analysis and investigation were conducted by J.-I.P. and D.-K.K. Reviews and editing were conducted by P.L., I.M.K., J.J., and H.K. Project administration was conducted by J.-H.B. All authors have read and agreed to the published version of the manuscript.

Funding: This research was funded by National Research Foundation of Korea (NRF) and ICT, 2018R1A2B6008815 and was funded by the Ministry of Education of Korea, 21A20131600011.

Acknowledgments: This research was supported by the Basic Science Research Program through the National Research Foundation of Korea (NRF) funded by the Ministry of Science and ICT (2018R1A2B6008815) and by the BK21 Plus project funded by the Ministry of Education, Korea (21A20131600011).

Conflicts of Interest: The authors declare no conflict of interest.

\section{References}

1. Ha, C.; Lee, H.J.; Kwon, J.W.; Seok, S.Y.; Ryoo, C.I.; Yun, K.Y.; Cha, S.Y. 69.2: Distinguished Paper: High Reliable a-IGZO TFTs with Self-Aligned Coplanar Structure for Large-Sized Ultrahigh-Definition OLED TV. Dig. Tech. Pap. 2015, 46, 1020-1022. [CrossRef]

2. Bae, J.U.; Kim, D.H.; Kim, K.; Jung, K.; Shin, W.; Kang, I.; Yeo, S. 10.2: Invited Paper: Development of Oxide TFT's Structures. Dig. Tech. Pap. 2013, 44, 89-92. [CrossRef]

3. Hayashi, R.; Sato, A.; Ofuji, M.; Abe, K.; Yabuta, H.; Sano, M.; Hosono, H. 42.1: Invited Paper: Improved Amorphous In-Ga-Zn-O TFTs. Dig. Tech. Pap. 2008, 39, 621-624. [CrossRef]

4. Lee, G.J.; Kim, J.; Kim, J.H.; Jeong, S.M.; Jang, J.E.; Jeong, J. High performance, transparent a-IGZO TFTs on a flexible thin glass substrate. Semicond. Sci. Technol. 2015, 29, 035003. [CrossRef]

5. Kamiya, T.; Nomura, K.; Hosono, H. Present status of amorphous In-Ga-Zn-O thin-film transistors. Sci. Technol. Adv. Mater. 2010, 11, 044305. [CrossRef] [PubMed]

6. Ryu, B.; Noh, H.K.; Choi, E.A.; Chang, K.J. O-vacancy as the origin of negative bias illumination stress instability in amorphous In-Ga-Zn-O thin film transistors. Appl. Phys. Lett. 2010, 97, 022108. [CrossRef]

7. Nahm, H.H.; Kim, Y.S.; Kim, D.H. Instability of amorphous oxide semiconductors via carrier-mediated structural transition between disorder and peroxide state. Phys. Status Solidi B 2012, 249, 1277-1281. [CrossRef] 
8. Kang, Y.; Ahn, B.D.; Song, J.H.; Mo, Y.G.; Nahm, H.H.; Han, S.; Jeong, J.K. Hydrogen Bistability as the Origin of Photo-Bias-Thermal Instabilities in Amorphous Oxide Semiconductors. Adv. Electron. Mater. 2015, 1, 1400006. [CrossRef]

9. Kamiya, T.; Nomura, K.; Hosono, H. Electronic structure of the amorphous oxide semiconductor a-InGaZnO4-x: Tauc-Lorentz optical model and origins of subgap states. Phys. Status Solidi A 2009, 206, 860-867. [CrossRef]

10. Hosono, H. Ionic amorphous oxide semiconductors: Material design, carrier transport, and device application. J. Non-Cryst. Solids 2006, 352, 851-858. [CrossRef]

11. Minami, T. Transparent conducting oxide semiconductors for transparent electrodes. Semicond. Sci. Technol. 2005, 20, S35. [CrossRef]

12. Nelson, E. Derivation of the Schrödinger equation from Newtonian mechanics. Phys. Rev. 1966, 150, 1079. [CrossRef]

13. Kim, Y.; Bae, M.; Kim, W.; Kong, D.; Jung, H.K.; Kim, H.; Kim, D.H. Amorphous InGaZnO thin-film transistors-Part I: Complete extraction of density of states over the full subband-gap energy range. IEEE Trans. Electron Devices 2012, 59, 2689-2698. [CrossRef]

14. Park, J.; Kwon, J.H.; Battaglini, N.; Lang, P.; Bae, J.H.; Kim, H. Importance of active layer positioning on gate electrode in organic thin-film transistors. Mol. Cryst. Liq. Cryst. 2018, 660, 72-78. [CrossRef]

15. Kim, Y.; Kim, S.; Kim, W.; Bae, M.; Jeong, H.K.; Kong, D.; Kim, D.H. Amorphous InGaZnO thin-film transistors-Part II: Modeling and simulation of negative bias illumination stress-induced instability. IEEE Trans. Electron Devices 2012, 59, 2699-2706. [CrossRef]

16. Fung, T.C.; Chuang, C.S.; Chen, C.; Abe, K.; Cottle, R.; Townsend, M.; Kanicki, J. Two-dimensional numerical simulation of radio frequency sputter amorphous In-Ga-Zn-O thin-film transistors. J. Appl. Phys. 2009, 106, 084511. [CrossRef]

17. Heiman, F.P.; Warfield, G. The effects of Oxide Traps on the MOS Capacitance. IEEE Trans. Electron Devices 1965, 12, 167-178. [CrossRef]

18. Altas User's Manual; Silvaco Inc.: Santa Clara, CA, USA, 2016.

19. Stewart, K.A.; Wager, J.F. Thin-film transistor mobility limits considerations. J. Soc. Inf. Disp. 2016, 24, 386-393. [CrossRef]

20. Hurkx, G.A.M.; Klaassen, D.B.M.; Knuvers, M.P.G.; O’hara, F.G. A new recombination model describing heavy-doping effects and low-temperature behaviour. In Proceedings of the International Technical Digest on Electron Devices Meeting, Washington, DC, USA, 3-6 December 1989; pp. 307-310.

21. Hu, C. Modern Semiconductor Devices for Integrated Circuits; International edition; Prentice Hall: Upper Saddle River, NJ, USA, 2009; pp. 56-63.

22. Li, L.; Lu, N.; Liu, M. Field effect mobility model in oxide semiconductor thin film transistors with arbitrary energy distribution of traps. IEEE Electron Device Lett. 2014, 35, 226-228. [CrossRef] 